\title{
A novel mutation in the COL2A1 gene in a patient with Stickler syndrome type 1: a case report and review of the literature
}

\author{
Yousuke Higuchi ${ }^{1,2}$, Kosei Hasegawa ${ }^{2 *}$, Miho Yamashita ${ }^{2,3}$, Hiroyuki Tanaka ${ }^{4}$ and Hirokazu Tsukahara ${ }^{1}$
}

\begin{abstract}
Background: Stickler syndrome is a group of collagenopathies characterized by ophthalmic, skeletal, and orofacial abnormalities, with the degree of symptoms varying among patients. Mutations in the COL2A1, COL11A1, and COL11A2 procollagen genes cause Stickler syndrome. Marshall syndrome, caused by a COL11A1 mutation, has clinical overlap with Stickler syndrome.

Case presentation: A 2-year-old Japanese boy was presented to our hospital with short stature $(79.1 \mathrm{~cm},-2.52$ standard deviation). His past medical history was significant for soft cleft palate and bilateral cataracts. He had a flat midface, micrognathia, and limitations in bilateral elbow flexion. Radiographs showed mild spondyloepiphyseal dysplasia. Initially, we suspected Marshall syndrome, but no mutation was identified in COL11A1. At 8 years old, his height was 116. $2 \mathrm{~cm}$ (-1.89 standard deviation), and his orofacial characteristics appeared unremarkable. We analyzed the COL2A1 gene and found a novel heterozygous mutation (c.1142 G > A, p.Gly381Asp).

Conclusions: In this case report, we identify a novel missense mutation in the COL2A1 gene in a patient with Stickler syndrome type 1, and we describe age-related changes in the clinical phenotype with regard to orofacial characteristics and height. Genetic analysis is helpful for the diagnosis of this clinically variable and genetically heterogeneous disorder.
\end{abstract}

Keywords: Stickler syndrome, COL2A1, Type II collagenopathy, Marshall syndrome

\section{Background}

Stickler syndrome is a group of hereditary conditions characterized by high myopia, retinal detachment, cataracts, sensorineural or conductive hearing loss, mild spondyloepiphyseal dysplasia, early-onset osteoarthritis, midfacial underdevelopment, and cleft palate (as part of the Pierre Robin sequence). The degree of these symptoms varies among individuals. The incidence of Stickler syndrome is estimated to be approximately 1 in 7500 9000 neonates [1]. Stickler syndrome is a genetically heterogeneous disorder caused by abnormal synthesis of type II, XI, or IX collagen. Stickler syndrome type 1 (STL1) [MIM:108300] is the most common type, caused by mutations in the COL2A1 [MIM:120140] gene on chromosome 12q13.11. Stickler syndrome type 2 [MIM:604841] is the second most common, caused by

\footnotetext{
* Correspondence: haseyan@md.okayama-u.ac.jp

${ }^{2}$ Department of Pediatrics, Okayama University Hospital, 2-5-1 Shikata-cho,

Kita-ku, Okayama 700-8558, Japan

Full list of author information is available at the end of the article
}

mutations in the COL11A1 gene [MIM:120280] on chromosome 1p21. Stickler syndrome type 3 (autosomal dominant otospondylomegaepiphyseal dysplasia)[MIM:184840] is caused by mutations in the COL11A2 gene [MIM:120290] on chromosome 6p21.3. Stickler syndrome type 3 presents with characteristics similar to those of STL1 and Stickler syndrome type 2, except for the lack of ocular manifestations. These three types of Stickler syndrome show autosomal dominant heredity. Autosomal recessive Stickler syndrome has been reported in rare cases with mutations in collagen IX genes [2]. COL2A1 encodes an alpha-1 chain procollagen monomer that assembles into a homotrimer. These stable triple helices aggregate into fibrillar collagen (type II collagen). Type XI collagen is also fibrillar collagen and regulates the fibril diameter of type II collagen. Type IX collagen is a fibril-associated collagen with interrupted triple helices. Types II, XI, and IX collagen are expressed in the same tissues, which include cartilage, vitreous humor, intervertebral discs, and the inner ear. 
Marshall syndrome [MIM:154780], which is caused by mutations in COL11A1, has a clinical overlap with Stickler syndrome [3]. Distinguishing between Stickler syndrome and Marshall syndrome is not easy. It has been suggested that people with Marshall syndrome more often have a short stature, early-onset hearing loss, and more pronounced maxillary hypoplasia [4-6]. In this report, we describe a case of a patient with STL1 with a novel mutation in the COL2A1 gene.

\section{Case presentation}

A 24-month-old Japanese boy was referred to our hospital for evaluation of his short stature. He was born at a gestational age of 38 weeks and 4 days and had a submucous cleft palate and micrognathia. His birth weight was $3225 \mathrm{~g}(+0.53$ standard deviation (SD) of average Japanese male infants), and his birth length was $45 \mathrm{~cm}(-1.80 \mathrm{SD})$. His submucous cleft palate was repaired at 18 months of age. Tympanostomy tubes were placed at the same time for secretory otitis media. Auditory brainstem response testing showed normal findings. At 22 months, he was found to have bilateral leukocoria. Phacoemulsification and anterior vitrectomy for bilateral lens opacities were performed at another hospital. $\mathrm{He}$ was considered to have Pierre Robin sequence, but the association with the ophthalmic symptoms was not investigated. He received speech therapy for speech delay.

At presentation, the patient weighed $11.6 \mathrm{~kg}(-0.33$ SD) with a height of $79.1 \mathrm{~cm}(-2.52 \mathrm{SD})$. His arm span was $79 \mathrm{~cm}$, and his sitting height was $48.5 \mathrm{~cm}$ (sitting height/height ratio 0.61 ). He showed ocular hypertelorism; prominent eyes due to shallow eye sockets; a flat midface with a depressed nasal bridge; anteverted nares; micrognathia; low-set ears; and limitations in bilateral elbow flexion; but no hypotonia, hyperextensible joints, or joint pain. All other family members, which included his parents and two brothers, were healthy. His parents are not consanguineous. His father's height was $182 \mathrm{~cm}$ $(+1.93$ SD of average Japanese adult men), and his mother's height was $147 \mathrm{~cm} \mathrm{(-2.09} \mathrm{SD} \mathrm{of} \mathrm{average} \mathrm{Japanese}$ adult women). Radiographs showed thickening of the calvaria, widening of the distal humeral metaphysis, lack of femoral head ossification, deformity of the femoral neck, and distal femoral and proximal tibial epiphyseal ossification centers. These features suggested mild spondyloepiphyseal dysplasia (Fig. 1a-d). Serum insulin-like growth factor-1 was $20.17 \mathrm{nmol} / \mathrm{L}(154 \mathrm{ng} / \mathrm{ml}$ ) (normal range 2.36-20.17 nmol/L [18-154 ng/ml]). An L-arginine stimulation test for growth hormone $(0.5 \mathrm{~g} / \mathrm{kg}$ arginine) showed a peak growth hormone level of $16.4 \mathrm{ng} / \mathrm{ml}$. A luteinizing hormone-releasing hormone/thyrotropin-releasing hormone stimulation test $(3 \mu \mathrm{g} / \mathrm{kg}$ luteinizing hormone-releasing hormone; $10 \mu \mathrm{g} / \mathrm{kg}$ thyrotropin-releasing hormone) showed prepubertal and normal thyroid-stimulating hormone responses. His karyotype was 46,XY. A brain magnetic resonance imaging scan showed no abnormalities. Echocardiography showed no evidence of mitral valve prolapse. At that time, we suspected Marshall syndrome, but no mutation was identified in exons or exon/intron boundaries of the COL11A1 gene.

At the age of 8 years, the patient's height was $116.2 \mathrm{~cm}$ (-1.89 SD) (Fig. 2), and his orofacial characteristics appeared unremarkable. Although 3D reformatted computed tomographic scans showed atlantoaxial dysplasia, he had no clinical symptoms of atlantoaxial instability (Fig. 1e and f). He developed no symptoms of retinal detachment, deafness, or osteoarthritis. The patient studied at a normal elementary school and his mental development was normal. We next suspected Stickler syndrome and conducted COL2A1 genetic analysis. Genomic deoxyribonucleic acid was extracted from the patient's peripheral blood. Polymerase chain reaction primers were designed to amplify all coding exons and exon/intron boundaries of the COL2A1 gene. Primer sequences are available upon request. Sequence analysis showed a novel heterozygous mutation in exon 19 of COL2A1, c.1142G > A (p.Gly381Asp) (Fig. 3a), and no other mutation was identified. The glycine at amino acid 381 was located in the Gly-X-Y triplet repeats region of the type II collagen triple helix, and it is phylogenetically conserved throughout species (Fig. 3b) [7]. This variant has not been described in the Single Nucleotide Polymorphism database, the 1000 Genomes Project, Leiden Open Variation Database 3.0, or the Human Genetic Variation Database in Japanese. In silico analysis showed that this mutation was predicted to be "probably damaging" by PolyPhen-2 (score 0.999) and to be "disease causing" by MutationTaster (score 94) $[8,9]$.

\section{Discussion}

In this case report, we identify a novel substitution of glycine within the Gly-X-Y triplet repeats region of the type II collagen triple helix. Authors of several studies have reported that most mutations that cause type II collagenopathy are distributed in the Gly-X-Y triplet repeats region, and there is no hot spot for mutations in the COL2A1 gene $[1,10,11]$. Glycine replacement within the Gly-X-Y repeat accounts for many of the mutations and confirms that this type of gene alteration predominates in type II collagenopathies $[10,11]$. The changes elicit structural alterations of half of the alpha- 1 chains and result in abnormal conformation and destabilization of the triple helix $[11,12]$. We therefore concluded that this mutation was responsible for this patient's phenotype.

Heterozygous mutations in the COL2A1 gene cause a spectrum of type II collagenopathies, including those that are lethal in neonates, such as achondrogenesis type II/hypochondrogenesis [MIM:200610], and nonlethal conditions, such as spondyloepiphyseal dysplasia congenital [MIM:183900]; spondyloperipheral dysplasia 


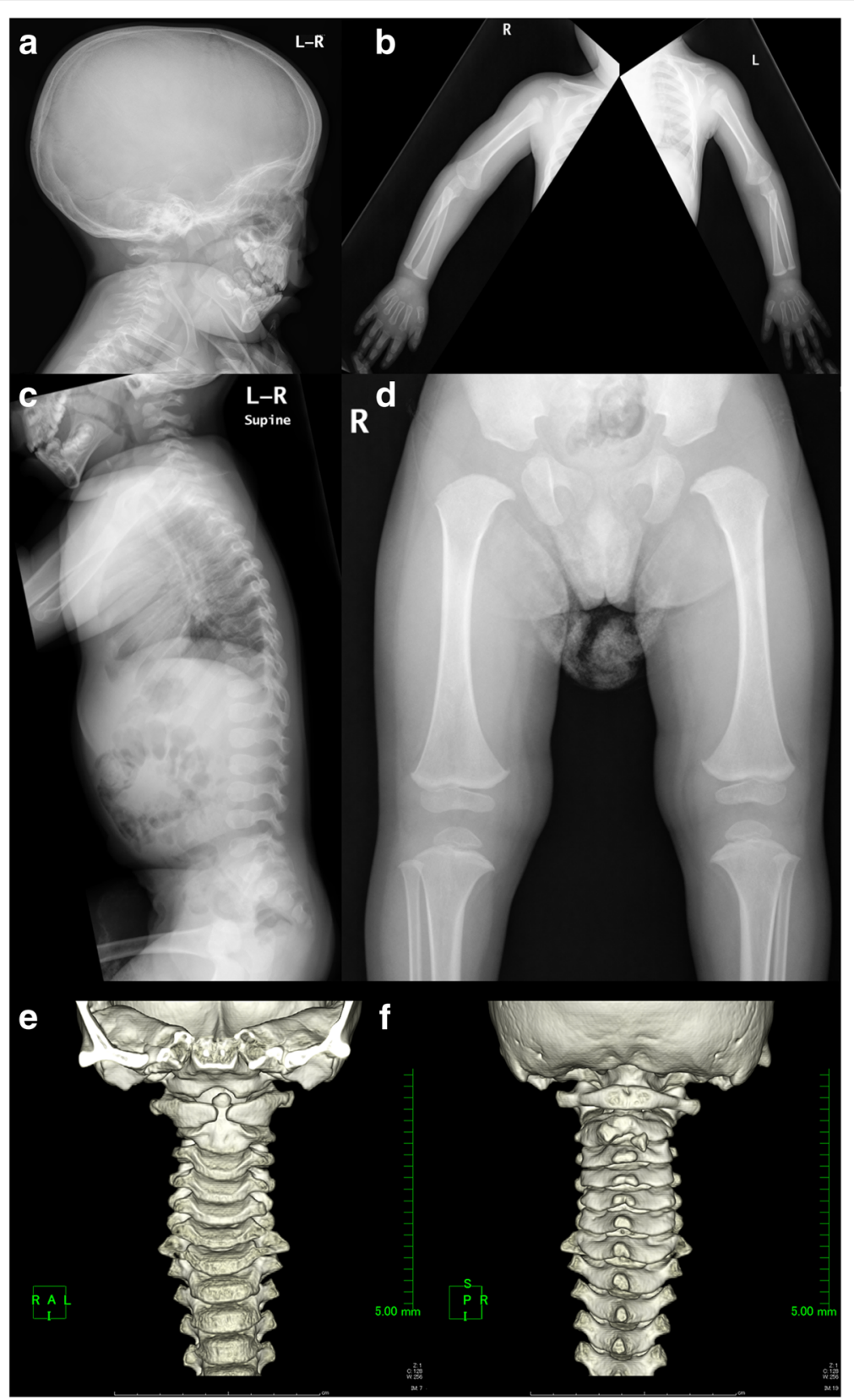

Fig. 1 Radiological images of the patient. Radiographic images show thickening of the calvaria (a); metaphyseal flaring of the distal humerus (b); mild kyphosis (c); and lack of femoral head ossification, deformity of the femoral neck, and distal femoral and proximal tibial epiphyseal ossification centers (d). Reformatted computed tomographic scans of the cenvical spine show hypoplasia of dens and separation of the anterior atlas arch (e), as well as abnormal ossification at the spinous process of the axis $(\mathbf{f})$

[MIM:271700]; spondyloepimetaphyseal dysplasia, Strudwick type [MIM:184250]; Kniest dysplasia [MIM:156550]; and Stickler syndrome. Ophthalmic, auditory, skeletal, and orofacial abnormalities are shared with these nonlethal type II collagenopathies; however, short trunk dwarfism is common in all but Stickler syndrome $[1,10]$. Our patient's short stature was mild and not disproportionate [13, 14]. Furthermore, orofacial features were not evident, and his short stature improved with age. Characteristics of midfacial underdevelopment in Stickler syndrome are more pronounced in infants and young children, and they become less distinctive with age $[15,16]$. In the majority of patients with Stickler syndrome, height is within the normal range, but the growth pattern is uncertain [17]. The phenotype of Stickler syndrome seems to become less distinct with increasing age, similar to quantitative or qualitative defects in type I collagen (for example, osteogenesis imperfecta) [18]. Premature stop (nonsense, 


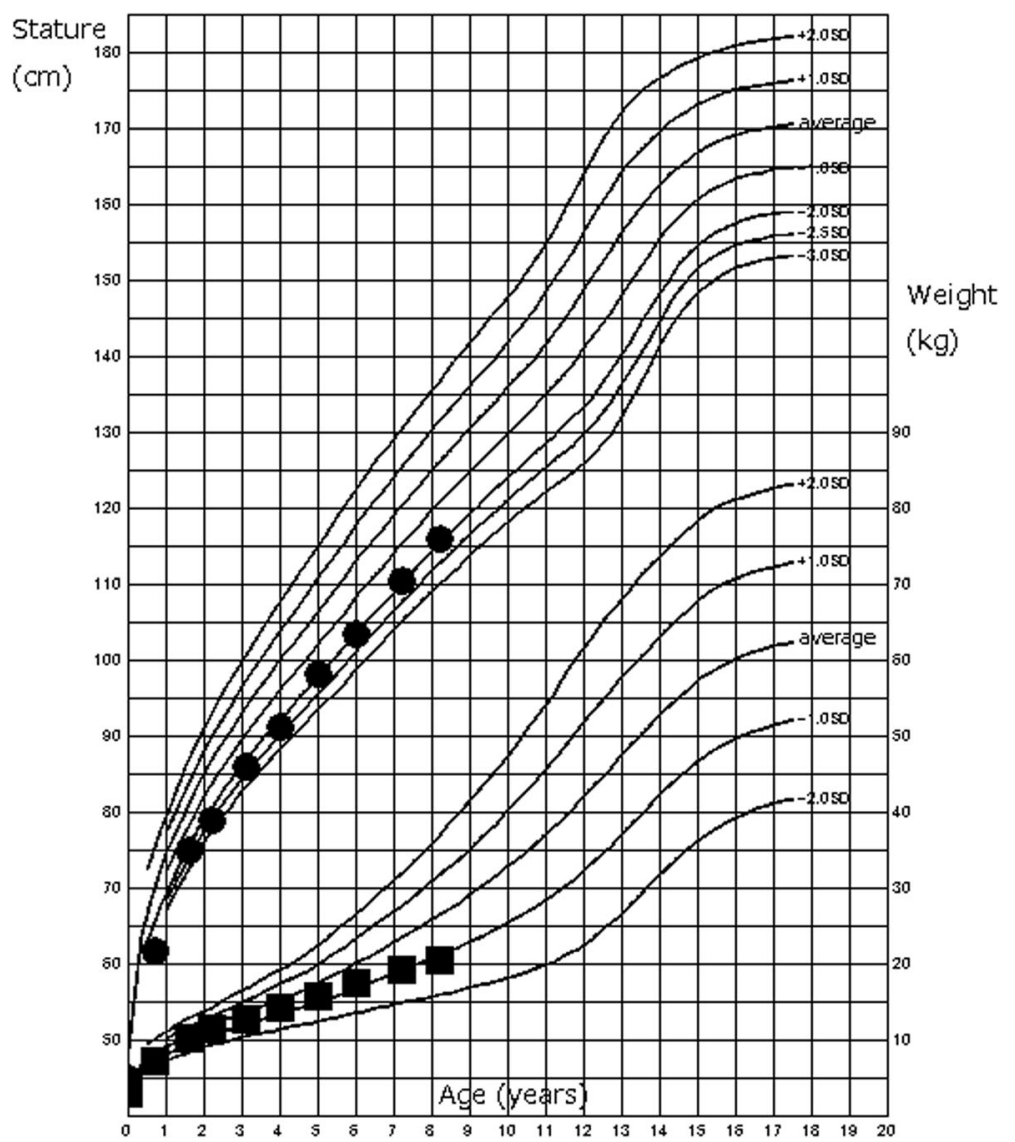

Fig. 2 Growth chart. The patient's short stature gradually improved over time

C A G G G IG A C C G GC C C C ACIGAIGCC C G TCG ICCTG A

a

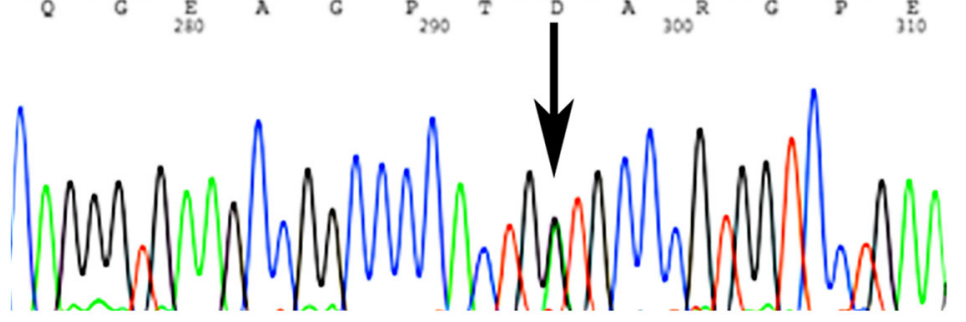

b

p.Gly381

Homo

Mus musculus Rattus

Gallus

Danio

Xenopus
GEAGPTGARGPEGAQ

GEAGPTGARGPEGAQ

GEAGPTGARGPEGAQ

GEAGPTGARGPEGAQ

GEAGPTGARGPEGAQ

GEAGPTGARGPEGAQ

Fig. 3 Mutation analysis of the COL2A1 gene. Heterozygous mutation c.1142G > A (p.Gly381Asp) is denoted by the arrow. The glycine substitution mutation is in the Gly-X-Y triplet repeats region of the type II collagen triple helix (a). The glycine at amino acid 381 is highly conserved throughout species (b) 
frameshift, or splicing) mutations of COL2A1 usually result in STL1, whereas glycine substitutions in the triplehelix region are typically associated with lethal (such as achondrogenesis type 2 /hypochondrogenesis) or more severe short stature (such as spondyloepiphyseal dysplasia congenital, Kniest dysplasia). The location of the glycine substitution or the nature of the substituting amino acid seems to affect the skeletal phenotype; however, there is no clear genotype-phenotype correlation $[1,10,11]$. On the basis of our clinical findings, the patient was diagnosed with STL1 [11].

Patients with Stickler syndrome exhibit varying signs and symptoms, which often may result in a delayed or missed diagnosis [17]. Some clinical diagnostic criteria for STL1 have been proposed, but they have not been validated $[1$, 19]. Authors of several studies have reported that Stickler syndrome is the basis for about $20-50 \%$ of infants with Pierre Robin sequence [20, 21]. It was suggested that Stickler syndrome should be considered in any neonates with Pierre Robin sequence, particularly in those with a family history of cleft palate and in patients with dominantly inherited myopia, nontraumatic retinal detachment, and/or mild spondyloepiphyseal dysplasia [22]. Accurate diagnosis is important for the assessment and management of ophthalmic, auditory, and articular manifestations, which are sometimes not obvious, and also for genetic counseling.

Marshall syndrome is similar to Stickler syndrome, and there has been debate over whether Stickler syndrome and Marshall syndrome are distinct conditions [4, 17, 23]. Genetic analysis has revealed that both diseases are allelic disorders caused by mutations in the COL11A1 gene $[5,6]$. Most patients with Marshall syndrome have a splice-site mutation in the COL11A1 gene [3]. A short nose, anteverted nares, midfacial hypoplasia, and a flat nasal bridge are common in patients with Marshall syndrome and are also seen in young patients with mutations in the COL2A1 gene (STL1) [5]. We first considered a diagnosis of Marshall syndrome, but molecular analysis confirmed a diagnosis of STL1.

\section{Conclusions}

We identified a novel missense mutation in the COL2A1 gene in a patient with STL1, and we report age-related changes in the clinical phenotype with regard to orofacial characteristics and height. Genetic analysis is helpful for the diagnosis of this clinically variable and genetically heterogeneous disorder.

\section{Abbreviation}

STL1: Stickler syndrome type 1

\section{Acknowledgements}

We thank Takuma Matsuyama (student in the Faculty of Medicine, Okayama University, Okayama, Japan) for carrying out the sequence analysis. We also thank Edanz (www.edanzediting.co.jp) for English-language writing assistance.

\section{Authors' contributions}

YH drafted the manuscript. KH carried out the clinical treatment and helped draft the manuscript. MY carried out the clinical treatment. HTa and HTs reviewed and finalized the manuscript. All authors read and approved the final manuscript.

\section{Ethics approval and consent to participate}

This study was approved by the institutional review board of Okayama University Hospital for clinical research. All procedures performed in studies involving human participants were done in accordance with the 1964 Helsinki declaration and its later amendments.

\section{Consent for publication}

Written informed consent was obtained from the patient's legal guardian(s) for publication of this case report and any accompanying images. A copy of the written consent is available for review by the Editor-in-Chief of this journal.

\section{Competing interests}

The authors declare that they have no competing interests.

\section{Publisher's Note}

Springer Nature remains neutral with regard to jurisdictional claims in published maps and institutional affiliations.

\section{Author details}

'Department of Pediatrics, Okayama University Graduate School of Medicine, Dentistry and Pharmaceutical Sciences, 2-5-1 Shikata-cho, Kita-ku, Okayama 700-8558, Japan. ${ }^{2}$ Department of Pediatrics, Okayama University Hospital, 2-5-1 Shikata-cho, Kita-ku, Okayama 700-8558, Japan. ${ }^{3}$ Faculty of Human Life Sciences, Notre Dame Seishin University, 9-16-2 Ifuku-cho, Okayama 700-8516, Japan. ${ }^{4}$ Department of Pediatrics, Okayama Saiseikai General Hospital, 1-7-18 Ifuku-cho, Kita-ku, Okayama 700-8511, Japan.

Received: 12 November 2016 Accepted: 20 July 2017

Published online: 26 August 2017

\section{References}

1. Hoornaert KP, Vereecke I, Dewinter C, Rosenberg T, Beemer FA, Leroy JG, et al. Stickler syndrome caused by COL2A1 mutations: genotype-phenotype correlation in a series of 100 patients. Eur J Hum Genet. 2010;18:872-80.

2. Faletra F, D'Adamo AP, Bruno I, Athanasakis E, Biskup S, Esposito L, et al. Autosomal recessive stickler syndrome due to a loss of function mutation in the COL9A3 gene. Am J Med Genet A. 2014;164:42-7.

3. Khalifa O, Imtiaz F, Ramzan K, Allam R, Al-Hemidan A, Faqeih E, et al. Marshall syndrome: further evidence of a distinct phenotypic entity and report of new findings. Am J Med Genet A. 2014;164:2601-6.

4. Aymé S, Preus M. The Marshall and Stickler syndromes: objective rejection of lumping. J Med Genet. 1984;21:34-8.

5. Annunen S, Körkkö J, Czarny M, Warman ML, Brunner HG, Kääriäinen H, et al. Splicing mutations of 54-bp exons in the COL11A1 gene cause Marshall syndrome, but other mutations cause overlapping Marshall/Stickler phenotypes. Am J Hum Genet. 1999;65:974-83.

6. Majava M, Hoornaert KP, Bartholdi D, Bouma MC, Bouman K, Carrera M, et al. A report on 10 new patients with heterozygous mutations in the COL11A1 gene and a review of genotype-phenotype correlations in type XI collagenopathies. Am J Med Genet A. 2007;143A:258-64.

7. Larkin MA, Blackshields G, Brown NP, Chenna R, McGettigan PA, McWilliam $H$, et al. Clustal W and Clustal X version 2.0. Bioinformatics. 2007:23:2947-8.

8. Adzhubei IA, Schmidt S, Peshkin L, Ramensky VE, Gerasimova A, Bork P, et al. A method and server for predicting damaging missense mutations. Nat Methods. 2010;7:248-9.

9. Schwarz JM, Rödelsperger C, Schuelke M, Seelow D. MutationTaster evaluates disease-causing potential of sequence alterations. Nat Methods. 2010;7:575-6.

10. Terhal PA, Nievelstein RJAJ, Verver EJJ, Topsakal V, van Dommelen P, Hoornaert $\mathrm{K}$, et al. A study of the clinical and radiological features in a cohort of 93 patients with a COL2A1 mutation causing spondyloepiphyseal dysplasia congenita or a related phenotype. Am J Med Genet A. 2015;167A:461-75.

11. Barat-Houari M, Sarrabay G, Gatinois V, Fabre A, Dumont B, Genevieve D, et al. Mutation update for COL2A1 gene variants associated with type II collagenopathies. Hum Mutat. 2016;37:7-15. 
12. Prockop DJ, Kivirikko Kl. Collagens: molecular biology, diseases, and potentials for therapy. Annu Rev Biochem. 1995;64:403-34.

13. Bogin B, Varela-Silva MI. Leg length, body proportion, and health: a review with a note on beauty. Int J Environ Res Public Health. 2010;7:1047-75.

14. Fredriks AM, van Buuren S, van Heel WJM, Dijkman-Neerincx RHM, VerlooveVanhorick SP, Wit JM. Nationwide age references for sitting height, leg length, and sitting height/height ratio, and their diagnostic value for disproportionate growth disorders. Arch Dis Child. 2005;90:807-12.

15. Temple IK. Stickler's syndrome. J Med Genet. 1989;26:119-26.

16. Snead MP, Yates JR. Clinical and Molecular genetics of Stickler syndrome. J Med Genet. 1999:36:353-9.

17. Stickler GB, Hughes W, Houchin P. Clinical features of hereditary progressive arthro-ophthalmopathy (Stickler syndrome): a survey. Genet Med. 2001;3:192-6.

18. Pereira RF, Hume EL, Halford KW, Prockop DJ. Bone fragility in transgenic mice expressing a mutated gene for type I procollagen (COL1A1) parallels the age-dependent phenotype of human osteogenesis imperfecta. J Bone Miner Res. 1995:10:1837-43.

19. Rose PS, Levy HP, Liberfarb RM, Davis J, Szymko-Bennett Y, Rubin Bl, et al. Stickler syndrome: clinical characteristics and diagnostic criteria. Am J Med Genet A. 2005;138A:199-207.

20. Izumi K, Konczal LL, Mitchell AL, Jones MC. Underlying genetic diagnosis of Pierre Robin sequence: retrospective chart review at two children's hospitals and a systematic literature review. J Pediatr. 2012;160:645-650.e2.

21. Antunes RB, Alonso N, Paula RG. Importance of early diagnosis of Stickler syndrome in newborns. J Plast Reconstr Aesthetic Surg. 2012;65:1029-34.

22. Jones KL. Marilyn Crandall Jones, Campo M del. Stickler syndrome. In: Jones $\mathrm{KL}$, editor. Smith's recognizable patterns of human malformation. 7th ed. Philadelphia: Saunders; 2013. p. 378-81.

23. Baraitser M. Marshall/Stickler syndrome. J Med Genet. 1982;19:139-40.

\section{Submit your next manuscript to BioMed Central and we will help you at every step:}

- We accept pre-submission inquiries

- Our selector tool helps you to find the most relevant journal

- We provide round the clock customer support

- Convenient online submission

- Thorough peer review

- Inclusion in PubMed and all major indexing services

- Maximum visibility for your research

Submit your manuscript at www.biomedcentral.com/submit

C) Biomed Central 